\title{
Erratum to: Racial disparities in early mortality in 1,134 young patients with acute stroke
}

\author{
Georgios Tsivgoulis · Jukka Putaala • Vijay K. Sharma · Clotilde Balucani $\cdot$ Sheryl Martin-Schild • \\ Sotirios Giannopoulos • Lokesh Batala • Christos Krogias • Paola Palazzo • Reza Bavarsad Shahripour • \\ Chrysa Arvaniti · Kristian Barlinn · Daniel Strbian • Elena Haapaniemi · Maria Flamouridou • \\ Konstantinos Vadikolias · Ioannis Heliopoulos • Konstantinos Voumvourakis • Nikos Triantafyllou • \\ Mahmoud Reza Azarpazhooh - Dimitrios Athanasiadis - Maria Kosmidou • Aristeidis H. Katsanos • \\ Spyros N. Vasdekis · Leonidas Stefanis • Elefterios Stamboulis • Charitomeni Piperidou • \\ Turgut Tatlisumak $\cdot$ Andrei V. Alexandrov
}

Published online: 20 February 2014

(c) Springer-Verlag Italia 2014

\section{Erratum to: Neurol Sci}

DOI 10.1007/s10072-014-1640-9

Unfortunately, the given name and family name of the coauthor Dr. Reza Bavarsad Shahripour were incorrectly published in the original publication. The correct given name and family name should read as 'Reza' and 'Bavarsad Shahripour', respectively.

The online version of the original article can be found under doi:10.1007/s10072-014-1640-9.

G. Tsivgoulis $(\bowtie) \cdot$ C. Arvaniti · L. Stefanis · E. Stamboulis Second Department of Neurology, School of Medicine,

"Attikon" Hospital, University of Athens, Iras 39, Gerakas

Attikis, 15344 Athens, Greece

e-mail: tsivgoulisgiorg@yahoo.gr

\section{G. Tsivgoulis}

International Clinical Research Center, St. Anne's University

Hospital Brno, Brno, Czech Republic

J. Putaala · D. Strbian · E. Haapaniemi - T. Tatlisumak Department of Neurology, Helsinki University Central Hospital, Helsinki, Finland

\section{K. Sharma}

Division of Neurology, Department of Medicine,

National University Hospital, Singapore, Singapore

\section{K. Sharma}

YLL School of Medicine, National University of Singapore,

Singapore, Singapore
C. Balucani - R. Bavarsad Shahripour · K. Barlinn ·

A. V. Alexandrov

Department of Neurology, Comprehensive Stroke Center,

University of Alabama at Birmingham, Birmingham, AL, USA

S. Martin-Schild

Stroke Program, Department of Neurology, Tulane University

Hospital, Tulane, New Orleans, LA, USA

S. Giannopoulos - M. Kosmidou - A. H. Katsanos

Department of Neurology, School of Medicine, University

of Ioannina, Ioannina, Greece

\section{Batala}

Department of Neurology, Narayana Medical College,

Nellore, Andhra Pradesh, India

C. Krogias

Department of Neurology, St. Josef-Hospital, Ruhr University, Bochum, Germany 


\section{P. Palazzo}

Neurologia Clinica, Università Campus Bio-Medico di Roma, Rome, Italy

R. Bavarsad Shahripour

Department of Neurology, Gholestan Hospital, Ahvaz University of Medical Sciences, Ahvaz, Iran

M. Flamouridou $\cdot$ K. Vadikolias $\cdot$ I. Heliopoulos

K. Voumvourakis - E. Stamboulis - C. Piperidou

Department of Neurology, School of Medicine, Democritus

University of Thrace, Alexandroupolis, Greece

\section{N. Triantafyllou}

First Department of Neurology, School of Medicine, "Eginition" Hospital, University of Athens, Athens, Greece

\section{R. Azarpazhooh}

Department of Neurology, Ghaem Hospital, Mashhad University of Medical Sciences, Mashhad, Iran

D. Athanasiadis · S. N. Vasdekis

Vascular Unit, 3rd Surgical Department, School of Medicine,

"Attikon" Hospital, University of Athens, Athens, Greece 